\title{
The Structure of the Human Yolk Sac: A Scanning and Transmission Electron Microscopic Analysis*
}

\author{
Jaime Pereda ${ }^{1}$, Silvia CorreR ${ }^{2}$ and Pietro M. Motta ${ }^{2}$ \\ Laboratorio de Embriologia y Microscopia Electronica ${ }^{1}$, Facultad de Medicina, Universidad de Chile, Santiago, Chile; and Istituto \\ di Anatomia Umana Normale², Facoltà di Medicina, Università di Roma "La Sapienza", Roma, Italy
}

Received December 8, 1993

Summary. Human yolk sacs were studied by light, transmission and scanning electron microscopy. Twelve human embryos at Carnegie stages ranging from 13 to 20 (28-49 days of gestation) were used for this research. The series of events which occur in the yolk sac wall during its period of maximum functional activity were recorded. The endodermal epithelium consisted of a single layer of columnar cells which, through cellular proliferation, formed endodermal cords which became cavitated, thereby forming endodermal vesicles. At the peak of yolk sac activity, intercellular spaces became very large and isolated individual endodermal cells. The epithelial cells were characterized by numerous microvilli on their free surface, high pinocytotic activity and by the formation of dense cisternae. Abundant intracellular vesicles fused together to empty their contents into the endodermal vesicles. The luminal surfaces of both intracellular and endodermal vesicles presented microvilli. The endodermal cells were characterized by an abundant granular endoplasmic reticulum, a welldeveloped Golgi apparatus, numerous mitochondria and glycogen particles. Endodermal vesicles were normally seen opening into the vitelline cavity through an endodermal orifice. The surface of the outer mesothelium was covered by numerous lengthy microvilli which were denser here than in the endodermal layer. A mucus-like material, present on the surface of the mesothelium, showed relatively few alterations during the study period. The mesothelial cells were less rich in organelles and far less active than the endodermal cells.

The microanatomy of the endoderm supports the contention that its cells serve as absorptive structures as well as sites of protein synthesis during early embryonic development. Therefore, the endodermal vesicles could function as a pump regulating the fluid volume into the vitelline cavity, thereby avoiding the collapse of the organ due to the absorptive activity of the endodermal cells. Furthermore, mesothelial microvilli together with their mucous material harbor a layer of serous exudate and thus create a lubricated cushion designed to protect the thin mesothelium from frictional damage.

During early human embryonic development, the definitive yolk sac (YS) is wedged into the exocoelomic cavity, remaining connected to the midgut by the narrow vitelline duct. As a consequence of the continuous growth of the amnios, the chorionic cavity narrows and the thin wall of the YS collapses against the chorion by the twelfth week of gestation. Several functions are attributed to the human YS. It is believed to be the initial hemopoietic organ of the embryo (BLOOM and BARTELEMEZ, 1940; FUKUDA, 1973; TAKASHINA, 1989), and the site of origin of primordial germ cells (WITSCHI, 1948). Furthermore, it performs an important role in the synthesis of various serum proteins (GITLIN and PERRICELLI, 1970; GiTLIN et al., 1972; SHI et al., 1985), and in exchange phenomena with the chorionic cavity.

Morphologically, while much is known about the structure of the YS in some mammalian species (DEMPSEY, 1953; King, 1971; TiedEMANN, 1976, 1977; KING and WILSON, 1983) very little is known regarding the YS architecture in humans. Few transmission electron microscopic studies of the human YS are found in the literature (HOYES, 1969; HESSELDAHL and LARSEN, 1969; FUKUDA, 1973; GonZALEZ-CRUSSI and RoTH, 1976; TAKASHINA, 1981), and scanning electron microscopy (SEM) investigations are also rare (UKESHIMA et al., 1986; PEREDA et al., 1987; TAKASHINA, 1989).

\footnotetext{
${ }^{*}$ Dr. J. PEREDA was a recipient of a bilateral research project from C. N. R. Italy and the University of Chile.

**Funds for this work were provided in part by MURST and CNR (Italy).
} 
Since the YS seems to be involved in many important functional activities that are crucial for early human embryonic development, we feel that its microanatomy deserves more definitive exploration in order to obtain a more accurate vision of its minute architecture. The microtopography and the functional purpose of the endodermal vesicles, as well as the functional role of the mesothelial surface during this period, have remained misunderstood. Obviously, a better knowledge of the anatomy of the YS wall may enhance understanding regarding the YS function.

This ultrastructural study, expanding on previous investigations (UKESHIMA et al., 1986; PEREDA et al., 1987), describes the microanatomy of the YS wall in human embryos at various stages of development. It focuses particularly on the development of the endodermal vesicular system and on the mesothelial layer.

\section{MATERIALS AND METHOD}

Eleven apparently normal YSs obtained from normal human embryos at 28, 30,33, 35, 42 and 49 days of gestation and corresponding to Carnegie Stages 13, 14, 15, 16, 17 and 20 (O'RAhilly and Muller, 1987) were analyzed in this study. The embryos were collected during salphingectomies performed on patients with ectopic tubal pregnancies. The ages of the embryos were determined from the date of the patient's last menstrual period; these were confirmed by a stereomicroscope according to anatomical characteristics and crown rump length.

The YS wall was examined by light microscopy (LM), transmission (TEM) and scanning electron microscopy (SEM). The YS were fixed by immersion in $3 \%$ glutaraldehyde in $0.1 \mathrm{M}$ phosphate buffer at $\mathrm{pH}$ 7.3 for $1 \mathrm{~h}$. For LM and TEM, the wall of the YSs were cut into small fragments. For SEM, specimens were cut longitudinally. Tissues were washed in the same buffer overnight and were post-fixed in $2 \%$ $\mathrm{OsO}_{4}$ in cacodylate buffer at $\mathrm{pH} 7.4$ at $4^{\circ} \mathrm{C}$, for $2 \mathrm{~h}$. After dehydration, specimens were embedded in Epon 812 for LM and TEM. The semithin sections for LM observation were stained with toluidine blue. The serial thin sections for TEM were stained according to REYNOLDS' method (1963), and examined with a Philips 301 TEM. For SEM, specimens were dehydrated in a graded series of ethanol and were critical point-dried with liquid $\mathrm{CO}_{2}$. Yolk sac samples were mounted on aluminium stubs, sputter coated with gold palladium, and observed with an ETEC Stereoscan and S-4000 Hitachi field emission SEM.

\section{RESULTS}

Fresh YSs, intact and normal in appearance, were collected. Their diameters were very similar, ranging from 4.5 to $5.0 \mathrm{~mm}$. YSs from embryos at Stage 13 were slightly oval, while older ones appeared pyriform and were covered with a mucous coat. They were connected to the embryos by a long, and narrow vitelline stalk. The vitelline wall in embryos at Stage 13 was $120-200 \mu \mathrm{m}$ thick. In contrast, the thickness of YSs derived from embryos at Stage 20 measured approximately 250 to $350 \mu \mathrm{m}$. This difference in thickness was probably due to the network of blood vessels present in old YSs.

As seen by LM and TEM, the thin wall of the YS consisted basically of three layers (Fig. 1): 1) an inner endodermal epithelium (EE) facing the YS cavity and continuous with the midgut epithelium;2) an outer mesothelial epithelium (ME) facing the exocoelomic cavity; and 3) an intermediate mesenchymal tissue interposed between the two epithelial surfaces.

\section{Endodermal epithelium}

LM showed the EE to be composed of a monolayer of cuboidal or columnar cells, independent of the functional stage of the YS. The large nuclei, containing one or two nucleoli, were located almost in the center of the cytoplasm together with numerous dark cytoplasmic bodies which were probably mitochondria. In YSs from embryos at Stage 13, the EE formed solid endodermal cords which projected deeply into the mesenchyme (Fig. 1). Serial semithin sections showed that most of these cords were cavitated (Fig. 2), forming endodermal vesicles (EVs) or so-called endodermal tubules (HESSELDAHL and LARSEN, 1969; TAKASHINA, 1981). The different types of EVs were always lined with radially arranged endodermal cells. In addition, they were frequently open by an endodermal pit on the side facing the vitelline cavity (Fig. 3). They were especially numerous around Stage 14.

By LM and TEM, the EE of YSs belonging to embryos at Stage 13 showed regularly distributed light and dark cells (Fig. 4). In contrast, only light endodermal cells were present in the EE of embryos from Stages 15 to 20.

In general, light endodermal cells were attached to a very fine basal lamina which was in close contact with the underlying mesenchymal tissue while, at the other pole, they were exposed to the vitelline cavity. The slightly convex surface of each cell presented numerous microvilli (Fig. 4).

The epithelial cells appeared uniformly outlined 


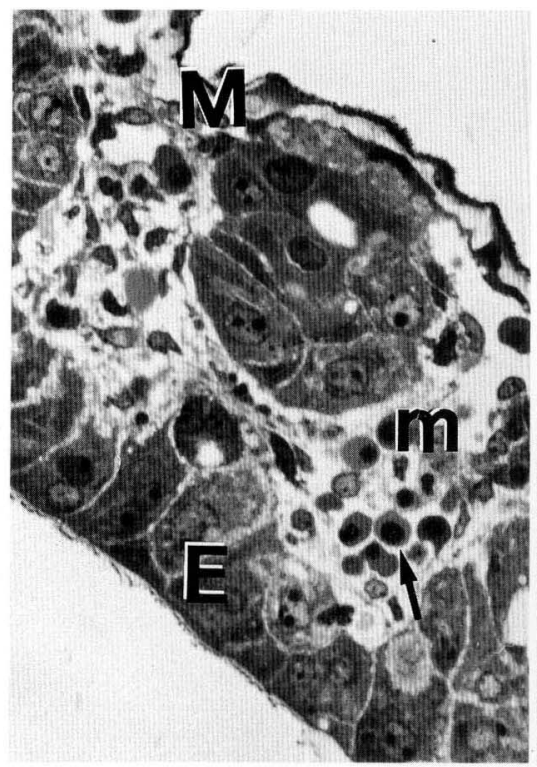

1

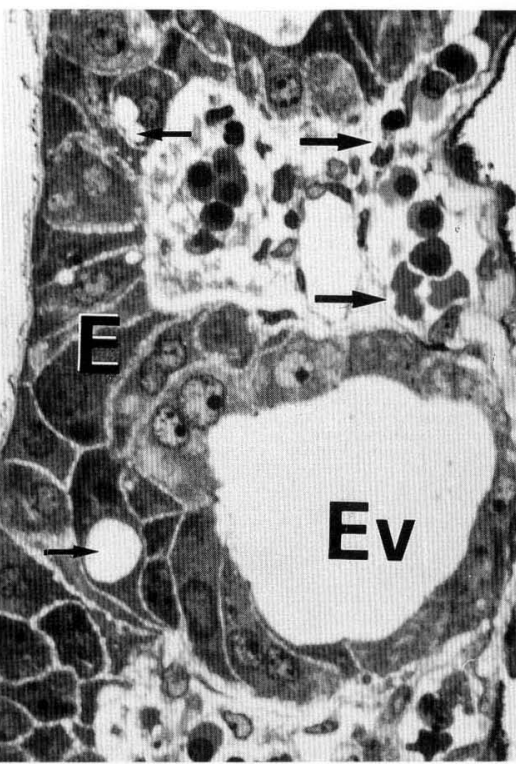

2

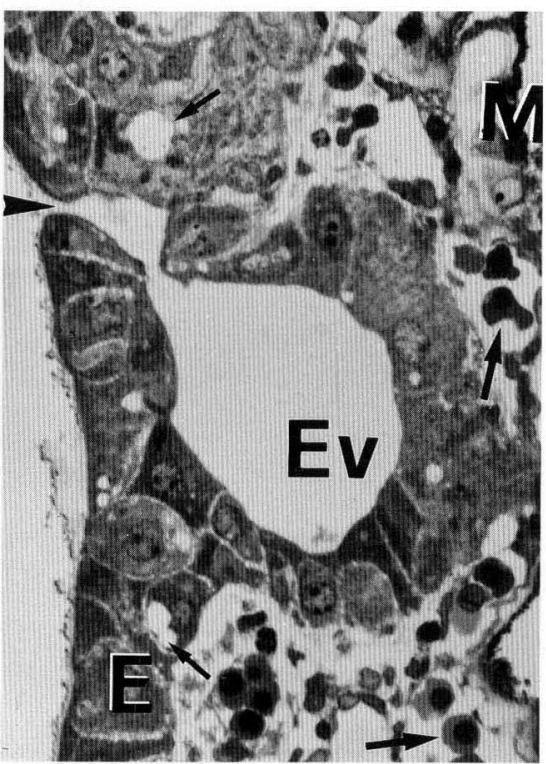

3

Figs. 1-3. Semithin sections stained with toluidine blue through the yolk sac wall showing the structural changes during the period of its functional activity. Fig. 1. General view of the yolk sac wall at Stage 13 as seen by LM. $E$ endodermal epithelium, $m$ mesechymal tissue layer, $M$ mesothelium, big arrow vitelline vessels. Fig. 2. Yolk sac wall at Stage 14. Note the endodermal cord $(E)$ and the endodermal vesicles $(E v)$ surrounded by mesenchymal tissue. The small arrows indicate intracellular vesicles in endodermal cells. Fig. 3. Yolk sac wall at Stage 14. An endodermal vesicle $(E v)$ is open to the yolk sac cavity through an endodermal pit (arrowheads). $M$ mesothelium. Figs. 1-3: $\times 370$

and were separated from each other by enlarged intercellular spaces (Fig. 5). The amplitude of these intercellular spaces was clearly evident in transversal sections through the endodermal cord from Stage 14 onward. Here, the endodermal cells were almost individually isolated (Fig. 6), remaining in close contact only by means of numerous microvilli which projected into the spaces. Regardless of the width of the intercellular spaces, none of these appeared to open directly into the vitelline cavity even though direct communication with the mesenchymal layer was noted (Fig. 7). Tight junctions between adjacent cells always occurred at the luminal surface of the epithelium (Fig. 5). Intracellular vesicles (ICVs) were frequently observed in the cortical cytoplasm of endodermal cells (Figs. 2, 3, 5).

By TEM, the cytoplasmic organelle distribution, as a rule, appeared in consistent patterns within endodermal cells (Figs. 4, 7) as follows: 1) The nuclear zone, which comprises the lower half of the cells, contained the nucleus. This was large and ovoid, often showing an irregular shape and possessing one or two nucleoli. Scattered Golgi cisternae, smooth vesicles and membranes of endoplasmic reticulum were located in the basal portion of the cells. 2) The

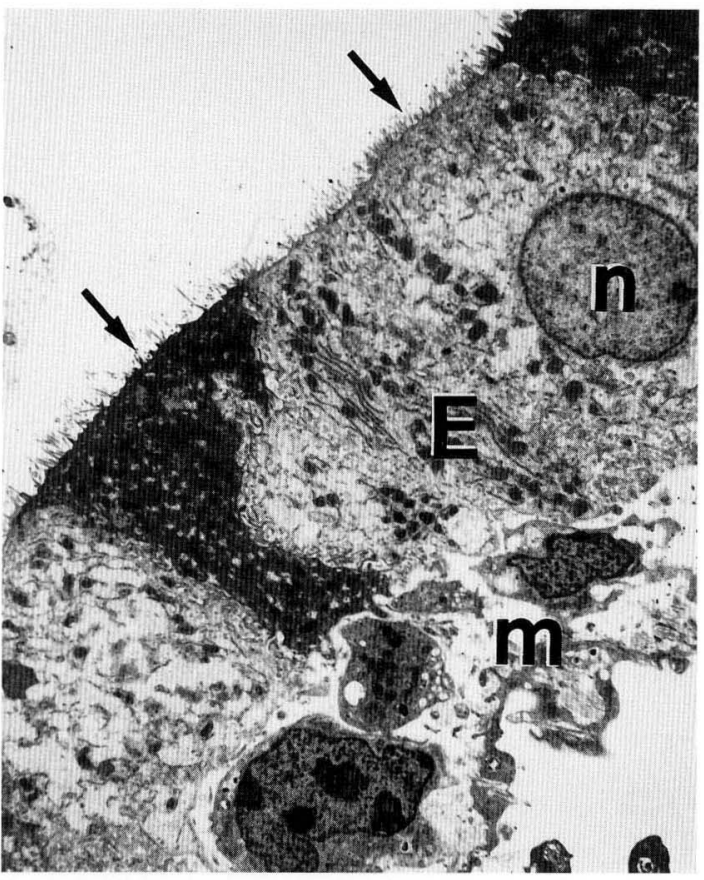

Fig. 4. Low magnification of the endodermal epithelium $(E)$ from a yolk sac at Stage 14 showing light and dark endodermal cells. Note the abundance of microvilli (arrows) at their surface. $m$ Mesenchymal tissue, $n$ nucleus. $\times 1,500$ 


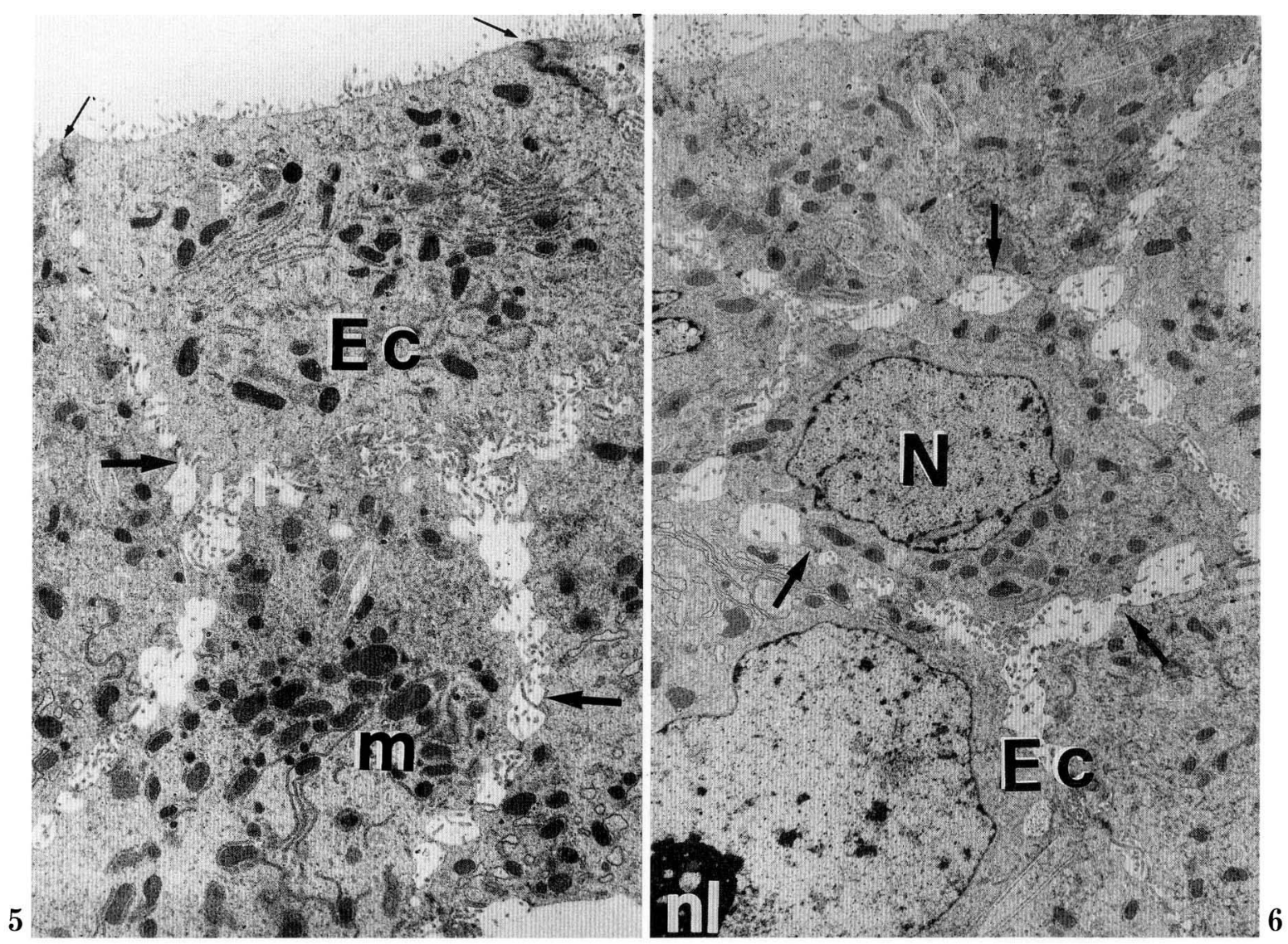

Fig. 5. Transmission electron microscopic image of part of the endodermal epithelium of the yolk sac wall at Stage 14. The structural relationship between endodermal cells $(E c)$ is shown. Note the well-developed intercellular spaces (big arrows) and the tight junctions (small arrows) at the cell neck. $m$ Mitochondria. $\times 5,000$

Fig. 6. A tangential section through a portion of the endodermal epithelium showing the isolation of the endodermal cell $\left(E_{c}\right)$ by large intercellular spaces (arrows). $N$ nucleus, $n l$ nucleolus. $\times 5,000$

cytoplasmic zones in the immediate vicinity of the intercellular spaces contained numerous dark and oval mitochondria with irregularly oriented cristae. They were usually associated with small vesicles of smooth endoplasmic reticulum (Figs. 5, 6). In addition, a tubular-cisternal complex was a distinctive characteristic of these zones (Figs. 5, 7). It consisted of tubular or flattened cisternal elements forming a belt around the middle and basal portions of the cells. The thick electron-dense wall of this membranous complex was well defined from the surrounding cytoplasm. 3) The apical zone was formed by a distinct fringe of microvilli located along the free edge of the cells and by a cortex containing fibrils of different thicknesses. This zona was crowded with a number of thin-walled vesicles, generally showing irregular shapes and a finely granular, homogeneous content. Coated vesicles which seemed to arise by pinocytosis from the free cell surface were also observed. Over- all, the contents of these organelles generally tended to be granular and heterogeneous. Furthermore, the organelles gave the cells a constant and distinct polarity even if they sometimes extended beyond their primary location into adjacent cellular areas. Finally, abundant glycogen granules were heterogeneously distributed in the cytoplasm.

The membranes of rough endoplasmic reticulum, which extended throughout the cell and consisted of several layers of flattened saccules arranged in a parallel manner, were particularly abundant near the nucleous and in the mitochondrial zones (Fig. 8), and frequently were continuous with the smooth endoplasmic reticulum. Microtubules were scattered throughout the cytoplasm and oriented in all directions. Free ribosomes, polysomes, and microfibrils were also observed.

By SEM, the endodermal surface appeared quite uniform in the different YSs analyzed. When viewed 
superiorly, much of the surface of the EE was covered with microvilli which were very regular in length and diameter and projected towards the vitelline cavity (Fig. 9). However, the distribution of microvilli per cell varied: they appeared abundant in the periphery and less numerous in the center of the apical cell surface. Furthermore, at the periphery of the cells, the microvilli were much longer (Fig. 10). Branched microvilli were not seen. The occurrence of pits on the epithelial surface (Fig. 11) confirms observations by LM. The pits were surrounded by several endodermal cells; these correspond to the orifices of endodermal vesicles (EVs). By TEM and SEM, the lumen of these EVs was lined with numerous microvilli (Fig. 11). A detailed analysis revealed that scattered cilia were also present among microvilli of regular length. The ICVs previously described by LM also showed that their luminal surface was populated by short microvilli. These vesicles varied in size; their contribution to the formation of EVs is shown in Figure 12. Normally, these ICVs opend into the EVs (Figs. 2, 3). As a rule, the size of the microvilli was consistent with the size of the ICV. Small ICVs had few and small microvilli. Neither blood cells nor other materials were revealed within the EVs by SEM. However, LM and TEM allowed frequent observation of a fine granular content.

By TEM, the cytoplasm of dark cells (Fig. 4) appeared as a very dense sap surrounding lacunae of varying sizes which contained a dense, granular material. The mitochondria of these cells were small and oval and displayed a dense matrix. Rough and smooth endoplasmic reticular membranes were abundant. The large, round nucleus exhibited a dense chromatin.

\section{Mesothelium}

By LM and TEM, the outer mesothelial layer was quite constant in its appearance throughout the period studied. The epithelial cells were usually flat or ovoid in shape with a very irregular free surface (Figs. 2, 3). This appearance was due to both the tendency of the cell bodies to protrude beyond the epithelial surface and to the abundance of large microvilli varying in thickness (Fig. 13). Their internal matrix consisted of low electron dense material which was continuous with the rest of the cell's matrix. Microvilli of different lengths, often flattened and branched (Fig. 13), were always longer than those of the endodermal cells. A different image of microvilli can be obtained by comparing TEM with SEM results. As seen in Figure 14, which shows a SEM panoramic view through a portion of the mesothelial surface, microvilli were usually uniform in their appearance. They

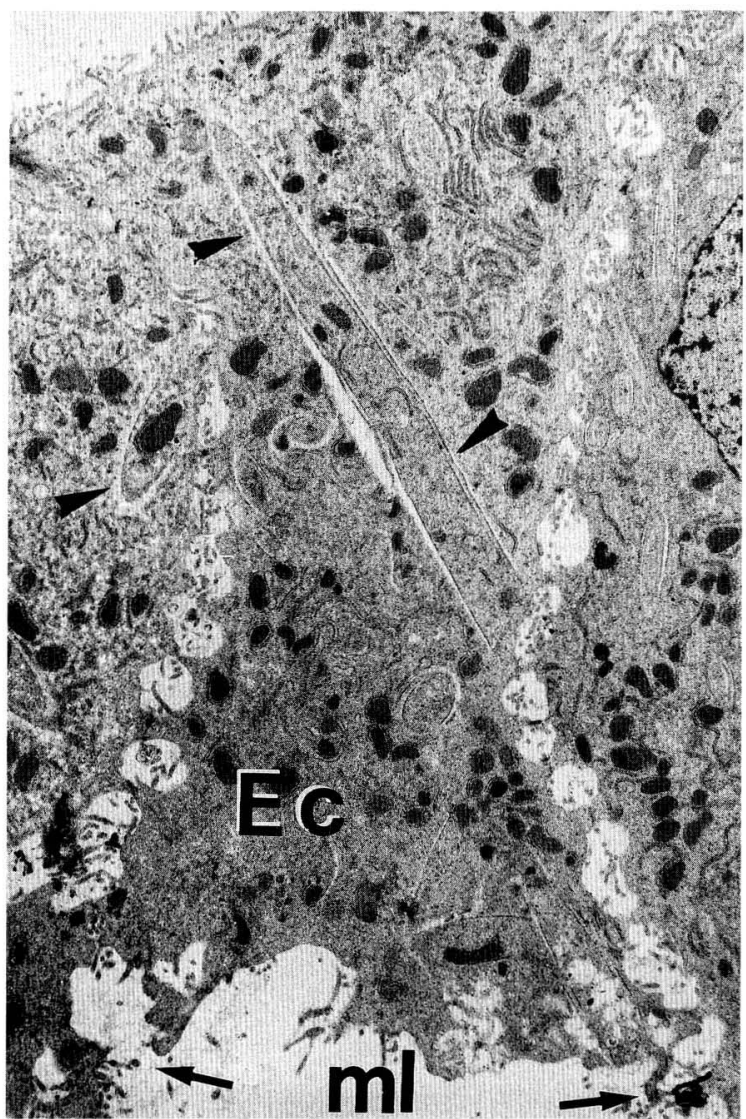

Fig. 7. TEM image of the endodermal cells $(E c)$. The apical edge of the cell presents numerous microvilli. Note that the intercellular spaces communicate directly with the underlying mesenchymal layer $(\mathrm{ml})$. arrowhead Tubular-cisternal complex. $\times 5,000$

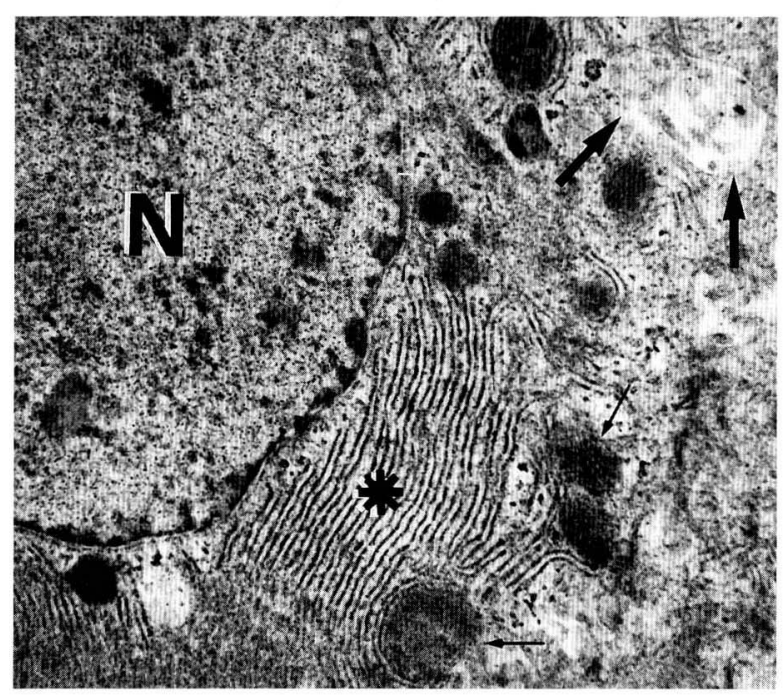

Fig. 8. TEM image of a portion of the endodermal cell showing a nucleus $(N)$, a prominent rough endoplasmic reticulum (asterix), round mitochondria (small arrow), and an intracellular vesicle (big arrows). $\times 15,000$ 


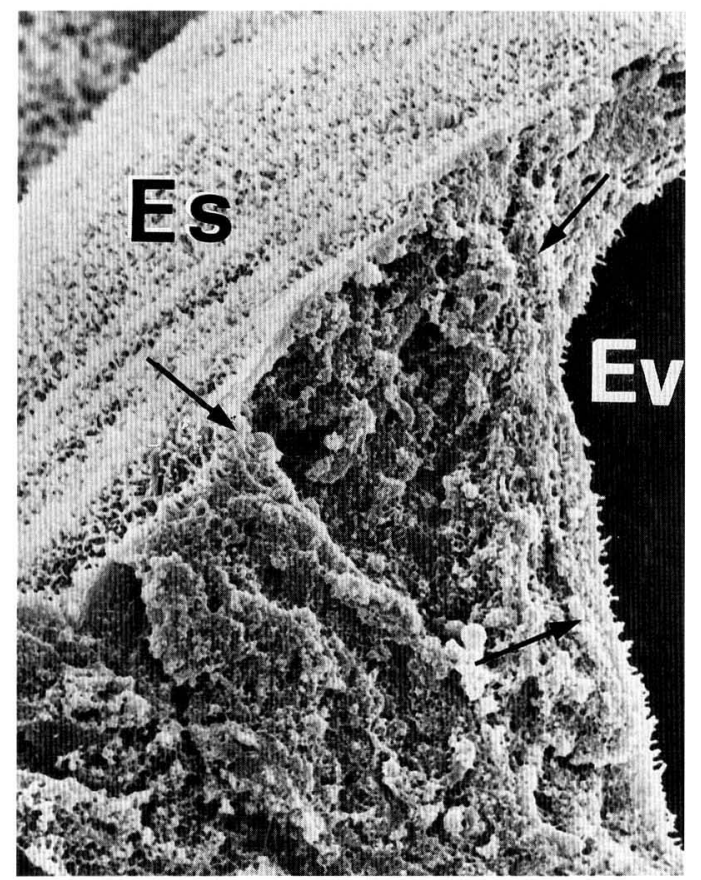

Fig. 9. SEM image of the wall of the yolk sac from an embryo at Stage 14 showing the endodermal surface $(E S)$, part of the endodermal cells and a portion of an endodermal vesicle $(E v)$. arrows Intercellular limits. $\times 3,000$

were very regular and constant in both their lengths and diameters.

TEM revealed the adjacent membranes of the intercellular space to be frequently tortuous or studded with short microvilli. Due to the flattened cell shape, the intercellular channels were much shorter than those of the EE and showed short terminal bars and also occasional desmosomes. On the basal surface, the mesothelium became increasingly irregular by Stage 20 and the cytoplasmic matrix became electron transparent due to the absence of organelles. A basal membrane was found at all stages. The nuclei were ovoid with peripheral heterochromatin. Smaller mitochondria were present in moderate numbers. A few coated vesicles and single membrane-bound vesicles were present near the apical surface. The rough endoplasmic reticulum, located throughout the cytoplasm of the mesothelial cells, was moderate in amount. No specific pattern was observed regarding cellular organelle distribution. At all stages studied, small collagen fibrils were often found in close association with the basal cell surface and sometimes in small pockets between the mesothelial cells.

From Stage 15 onwards, SEM showed a rugged appearence with irregular furrow-like depressions of

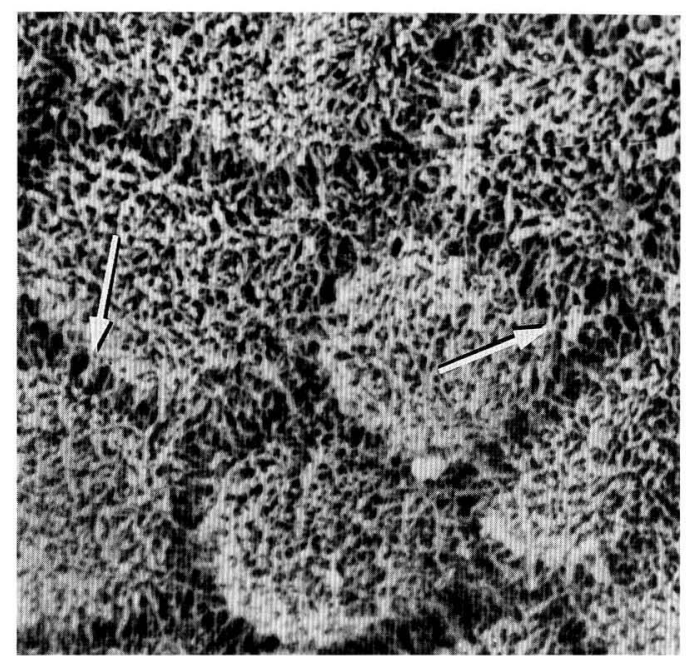

Fig. 10. A highly magnified SEM micrograph illustrating microvillous distribution on the surface of the endodermal cells (arrows). $\times 6,000$

various depths on the mesothelial surface of YSs. These features were likely due to the presence of a very rich network of blood vessels in the underlying mesenchyme. The rugged aspect became increasingly more pronounced as embryonic development progressed, peaking at around Stage 20. The mesothelial cells located on the surface of the blood vessels were different in size and shape from those present in other areas of the YS. They were flattened with very clear cellular limits, especially in older YSs. Their microvilli were less numerous than those of cells located in other regions. In all stages analyzed, a mucus-like material covered the mesothelial surface, leaving areas of mucus-free microvilli (Fig. 15).

\section{Mesenchymal tissue}

The most conspicuous feature of this tissue was its spongy-like appearance due to the presence of large spaces between adjacent mesenchymal cells and to blood vessels filled with numerous primitive erythroblasts (Fig. 16). Mesenchymal cells were the most abundant elements among fibers and other components of the extracellular matrix. They were stellate as a rule, even though other spindle-shaped cells were also present. Both types of cells formed major and minor processes which extended through the enveloping meshwork of the extracellular fibrous matrix (Fig. 17). The thickness of this layer depended upon the degree of blood vessel proliferation which occurred during early embryonal development. Occasionally, free macrophages were present in the mesen- 


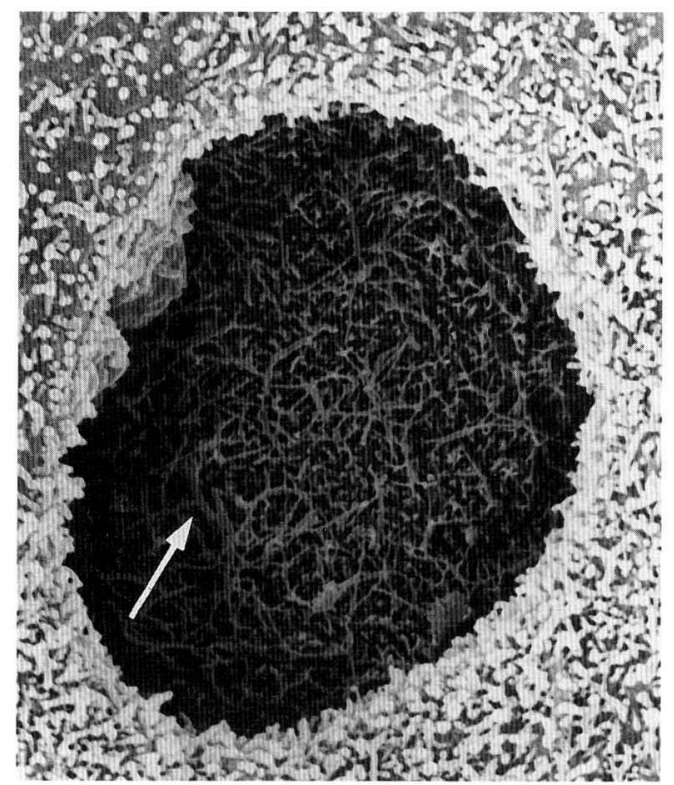

Fig. 11. SEM micrograph illustrating an endodermal orifice. Note the abundance of microvilli at the endodermal surface and inside the endodermal vesicles (arrow). $\times 4,500$

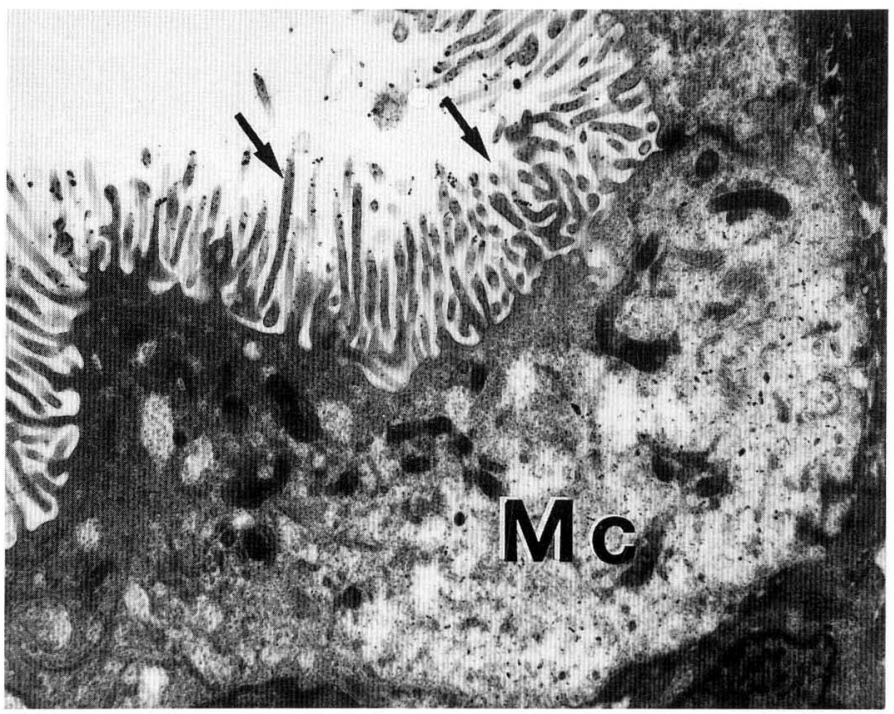

Fig. 13. A TEM micrograph of the mesothelial cell at Stage 15 $(M c)$. The numerous microvilli (arrows) are much longer than those in endodermal cells. $\times 8,000$

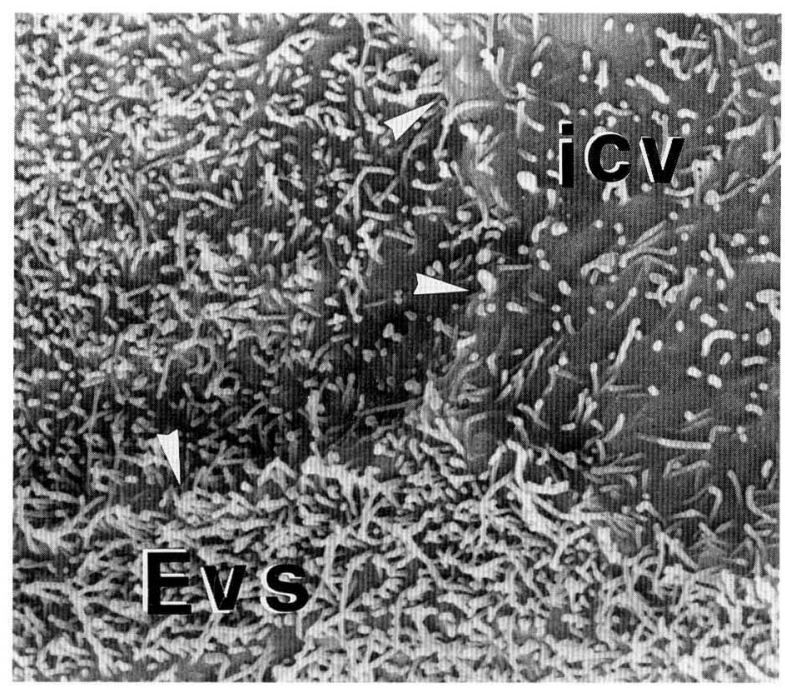

Fig. 12. Inside view of the endodermal vesicle surface (Evs) showing the outlines of parts of two intracellular vesicles ( $i c v)$ opening into the lumen of the endodermal vesicles (arrowheads). Note the differences in length and number among microvilli. $\times 6,000$

chymal tissue; these adhered to the surface of the blood vessels. Using SEM, they were identified by their voluminous cell body $(12-20 \mu \mathrm{m}$ in diameter) and by their numerous and slender cytoplasmic processes.

\section{DISCUSSION}

The YSs described in this report were obtained from human embryos which correspond to the morphological criteria listed by O'RAHILLY and MULLER (1987) for Stage 13, 14, 15, 16, 17 and 20, and presumably fall within the period of maximum functional activity (HOYEs, 1969). Both are important considerations if one wishes to correlate morphological and functional findings with those from other laboratories.

As is well known, the human YS develops as an extraembryonic organ close to the embryonic pole in the early weeks of gestation. However, the YS has fulfilled its function by the eleventh week and thereafter regresses as it becomes compressed by the growing amniotic sac. There is general agreement that the EE is the primary functional layer of the YS and that it performs functional activities which are crucial for early embryonic development (HESSELDAHL and LARSEN, 1969; FUKUDA, 1973; UKESHIMA et al., 1986; TAKASHINA, 1989). Nonetheless, various opinions still exist regarding the interpretation of the microanatomical data related not only to the EE (TAKASHINA, 1989), but also to the mesothelial layer (HESSELDAHL and LARSEN, 1969; FukUdA, 1973; UkEshima et al., 1986). These different interpretations might depend upon: 1) the stage of preservation of the specimens at the time the studies were carried out; 2 ) the criteria used to define 

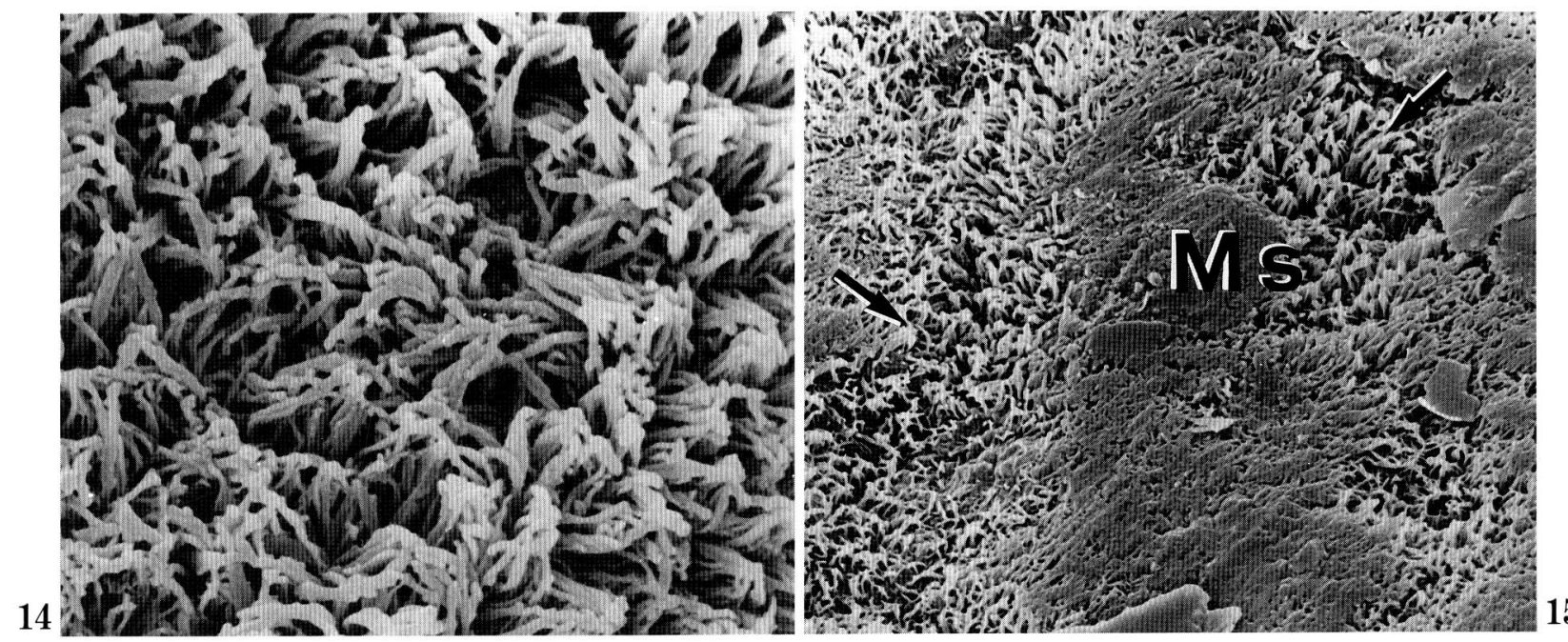

Fig. 14. SEM highly magnified microphotograph showing the free mesothelial surface covered by numerous and large microvilli. $\times 7,500$

Fig. 15. A general view of the mesothelial surface $(M S)$ of a YS from an embryo at Stage 16 . Note that a mucus-like material normally covers a large area of their surface. arrows Microvilli. $\times 2,700$
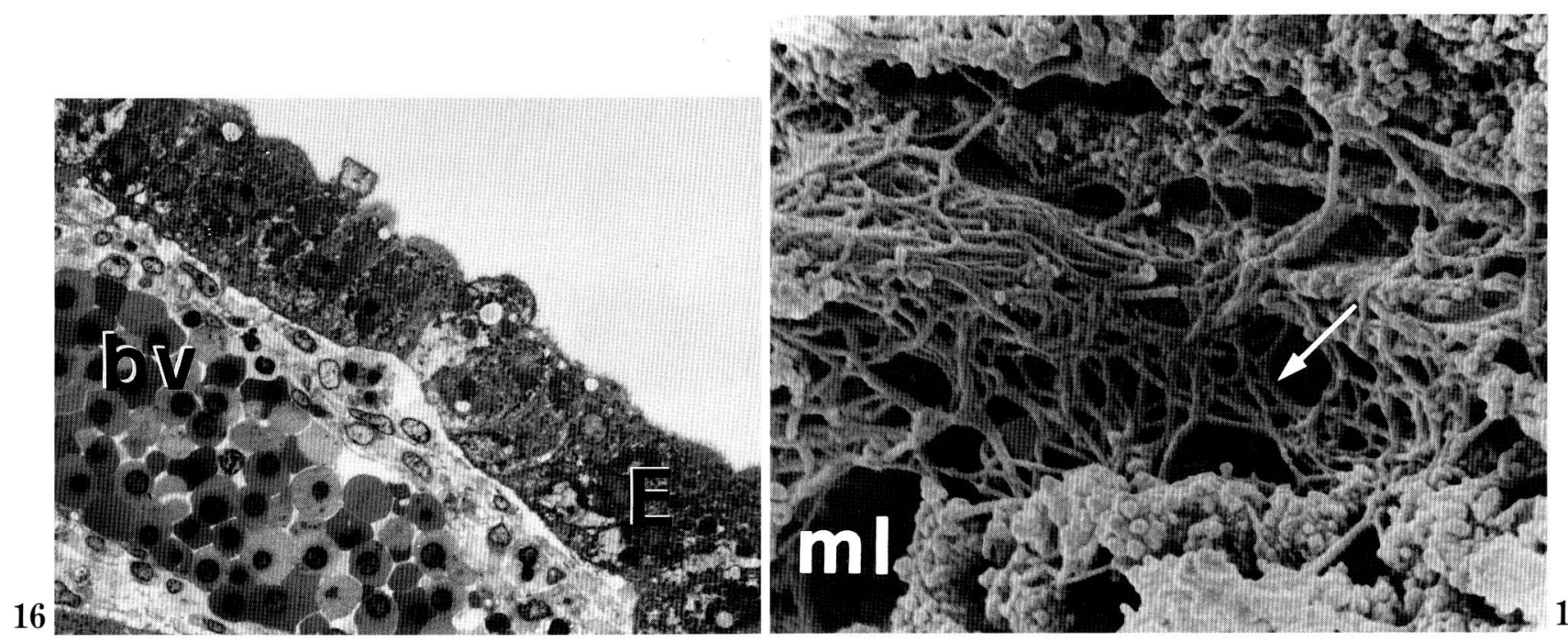

Fig. 16. LM picture of the yolk sac endodermal wall $(E)$ showing a blood vessel $(b v)$ in the mesenchymal layer containing numerous immature blood elements. $\times 400$

Fig. 17. SEM picture showing the arrangement of the fibers (arrow) in the mesenchymal layer $(m l)$ of a yolk sac from an embryo at Stage $16 . \times 9,000$

embryo normalcy; and 3) the technology used.

Correlated SEM and TEM studies, in addition to the LM serial analysis of the wall of the human YS, have not been used in depth: to our knowledge, only two reports are found in the literature (PEREDA et al., 1987; Ukeshima et al., 1989).

The wall of the YS is approximately constant in thickness throughout pregnancy. The endoderm is the most conspicuous tissue which occupies the major part of the wall during the early stages, while the mesenchyme becomes conspicuous as pregnancy proceeds. Initially, the endodermal layer consists of an endodermal epithelium facing the vitelline cavity. However, as development progresses, solid endodermal cords are produced with later become cavitated, thereby forming endodermal vesicles referred in the literature as endodermal tubules (HESSELDAHL and LARSEN, 1969; TAKASHINA, 1981). TAKASHINA (1989) 
indicated that these endodermal vesicles originated from the mesenchyme. According to our results, we could not find any evidence of a mesenchymal origin for EVs. However, our data clearly indicated that ICVs were at the origin of EVs. In fact, in the present work, we observed that ICVs contributed by exocitosis to the formation of EVs, and that large EVs arose by the fusion of small EVs. In order to understand the role of these EVs in YS function, it is necessary to consider the following points: The first refers to endodermal cell involvement in the absorption of substances from the vitelline cavity. This role can be deduced from the cellular architecture of the endodermal layer (HESSELDAHL and LARSEN, 1969). This is in fact characterized by columnar cells with large nuclei, the homogeneous distribution of microvilli forming a brush border-like structure at their free surface, and by the presence of numerous mitochondria, abundant endoplasmic reticulum, and a large number of different vesicles in their cytoplasm. These features, which were also observed in our results, are normally found in absorptive cells of the mammalian intestine (MотTA et al., 1977) and the visceral YSs of the guinea pig and rat embryos (DEMPSEY, 1953; WISLOCKI and DEMPSEY, 1955). Furthermore, the large intercellular spaces separating endodermal cells described in the current work are additional features that reinforce the idea that endodermal cells perform an absorptive activity (DEMPSEY, 1953; WISLOCKI and DEMPSEY, 1955; TiEDEMANN, 1976). This swelling effect of the intercellular spaces could be the result of fluid accumulation, however, we never observed any kind of material within this canalicular system. The fact that the actual transfer of molecules occurs from the endodermal epithelial cells to the mesenchymal compartment via the extracellular space is suggested by the polarization of cell organelles, including the Golgi apparatus and vesicles, to the canalicular side of the cells. The interruption of basal lamina continuity in these areas, as observed in the present study, may contribute to facilitate the intercellular transport into the mesenchymal tissue.

The second point for understanding the role of these EVs refers to the observation that the human YS in the exocoelomic cavity is essentially blown-up like a balloon by a serous fluid whose origin is still unknown. This fluid content seems to be responsible for the turgid characteristic of the organ. Therefore, we deduce that a constant intraluminal fluid pressure probably exists within the YS. As of now, we are unable to explain the mechanism involved in the regulation of YS fluid content and pressure. However, we believe that the maintenance of YSs intraluminal pressure during early embryogenesis is of paramount importance, not only for the morphogenetic process which gives rise to a normal embryo but also for the establishment of normal vitelline blood circulation. Therefore, if an active absorption of fluid from the vitelline cavity via microvilli occurs (HESSELDAHL and LARSEN, 1969), as seems to be the case, it is reasonable to assume that vitelline fluid pressure decreases as a consequence. Thus, the folding or the collapse of the vitelline wall into the lumen of the YS could occur if the fluid pressure is not reestablished in turn. At present, we are not certain if a flux of fluid occurs from the human intestinal tract to the vitelline cavity. Such an intestinal fluid contribution is probably unlikely if we consider that the lumen of the intestinal tract is obliterated at different levels by epithelial proliferation up to the 7 th week of gestation (OTANI et al., 1993).

Cosidering the previous arguments it seems reasonable to propose that ICVs and their neighboring EVs form a highly dynamic complex capable of rapid functional changes in response to the fluid modification ocurring within the lumen of YS. These changes, expressed as an excretory activity of the EVs towards the vitelline cavity via the endodermal orifice, might be the way by which the EVs control the fluid volume and pressure inside the YS during early development, thereby avoiding the folding or collapse of the YS wall. In other terms, in addition to its nutritional role, the EVs complex might also function as an hydraulic system in the regulation of the vitelline fluid pressure, rather than serving as the route for the transit of blood cells to the embryo via the vitelline cavity (TAKASHINA, 1989). We suggest that the excretion of fluid by the EVs could be stimulated by the reduction of the hydrostatic pressure inside the vitelline sac in order to maintain constant vitelline fluid pressure. Absorption, synthesis, secretion and removal of fluid must be regulated. We are presently unaware as to which mechanism is involved in the regulation of YS fluid content. TAKASHINA (1989) has suggested that EVs may play a role in the transport of erythroblasts. He based his interpretation on the observation of erythroblasts in the endodermal tubules of YS of embryos at the 5th week of pregnancy, which corresponds approximately to Stage 13. In the present work using similar techniques and embryos of almost identical ages as those shown in the figures by TAKASHINA (1989), we never saw any free blood cells passing into the endodermal vesicular system. Numerous blood vessels containing blood elements were always observed in the mesenchymal layer in proximity to the EVs. Occasionally, blood cells within the EVs and/or vitelline cavity were seen as the consequence of hemorrhage caused by mechan- 
ical damage during the collection or processing of embryos.

While much is known about the structure and possible function of endodermal cells, very scarce information is available on the role that mesothelial cells play in YS function. The mesothelial layer is part of the intraembryonic peritoneal layer with which it further shares a similar embryological origin. A very important characteristic of the peritoneal layer covering the surface of different organs within the body cavity is the presence, on its free surface, of a great amount of large and branched microvilli (ODOR, 1954; ANDREwS and PORTER, 1973; BARBERINI et al., 1988). It has been suggested that this rich microvillous covering is used by cells to increase their physiologically active areas. Since these cells are also normally covered by a serous exudate, it has been proposed that the different organs can be protected by this lubricating film against frictional damage or destruction. By virtue of its position within the exocoelom, it has been suggested that the YS is not likely involved in any major way in maternal-fetal exchange activities throughout the mesothelium. Rather significant information is presented in the current work to support the notion that YS mesothelium could perform a protective role similar to that played by the peritoneal lining. It is known that the human YS increases its size very little during early development, reaching a final diameter of 4.5 to 5.0 $\mathrm{mm}$ by the sixth week. During the same period, the amnios and the chorion both grow faster than the YS, while the length of the embryo increases. Consequently, the space within the exocoelomic cavity is reduced considerably. These phenomena probably expose the YS wall to erosion or compression which could produce early and irreversible damage to its structure and might therefore critically affect the normal development of the embryo. We believe that the rich microvillous border of mesothelial cells and the mucus-like material covering its surface may play an important lubricating role. This, in turn, may serve to protect the YS against adhesions and/or damage from compression, abrasion and other microenvironmental factors occurring during growth. By this mechanism, both premature deterioration and degeneration of the YS could be avoided.

In conclusion, this study illustrates the importance of the microanatomical analysis of the human YS by SEM and TEM since the quality of the information obtained by these methods improves our knowledge regarding its physiological role during early development. Many aspects of the YS function remain unresolved and its role in exchange phenomena with embryo through the vitelline duct has not been fully explored. Further research in this field is still necessary.

Acknowledgements. We wish to express our gratitude to Miss E. Vergnano for typing the manuscript and to Mr. Franchitto and Mr. Pozo for their skilful technical assistance.

\section{REFERENCES}

Andrews, P. M. and K. R. Porter: The ultrastructure morphology and possible functional significance of mesothelial microvilli. Anat. Rec. 177: 409-426 (1973).

Barberini, F., S. Correr and P. M. Motta: The peritoneum. In: (ed. by) P. M. MotTA and H. Fujita: Ultrastructure of the digestive tract. Martinus Njihoff Publishing, Boston, 1988. (p. 243-259)

Bloom, W. and G. W. BARTelemez: Hematopoiesis in young human embryos. Amer. J. Anat. 67: 21-53 (1940).

DEMPSEY, E. W.: Electron microscopy of the visceral yolk sac epithelium of the guinea pig. Amer. J. Anat. 93: 331-364 (1953).

FukudA, T.: Fetal hemopoiesis. Electron microscopic studies on human yolk sac hemopoiesis. Virchows Arch. (Cell Pathol.) 14B: 197-213 (1973).

Gituin, D. and A. Perricelli: Synthesis of serum albumin, prealbumin, a-fetoprotein, antitrypsin and transferrin by the human yolk sac. Nature 228: 995-997 (1970).

Gitlin, D., A. Perricelli and G. M. Gitlin : Synthesis of a-fetoprotein by liver, yolk sac, and gastrointestinal tract of the human conceptus. Cancer Res. 32: 979-982 (1972).

Gonzalez-Crussi, F. and L. M. Roth: The human yolk sac and yolk sac carcinoma. An ultrastructural study. Human Pathol. 7: 675-691 (1976).

Hesseldahl, H. and J. F. LARSEN: Ultrastructure of human yolk sac: endoderm, mesenchyme, tubules and mesothelium. Amer. J. Anat. 126: 315-336 (1969).

Hoyes, A. D.: The human fetal yolk sac. An ultrastructural study of four specimens. Z. Zellforsch. 99: 469-490 (1969).

KING, B. F.: Differentiation of parietal endoderm cells of the guinea pig yolk sac, with particular reference to the development of endoplasmic reticulum. Devel. Biol. 26: 547-559 (1971).

KING, B. F. and J. M. Wilson: A fine structural and cytochemical study of the rhesus monkey yolk sac: endoderm and mesothelium. Anat. Rec. 205: 143-158 (1983).

Motta, P. M., P. M. Andrews and K. R. Porter: Microanatomy of cell and tissue surfaces. An atlas of scanning electron microscopy. Lea \& Febiger, Philadelphia, 1977

ODOR, D. L.: Observations of the rat mesothelium with the electron and phase microscopes. Amer. J. Anat. 95: 433 (1954). 
O'RAhilly, R. and F. Muller: Developmental stages in human embryos. Carnegie Institution of Washington, Publication No. 637, 1986.

Otani, H., T. Yoneyama, R. Hashimoto, T. Hatta and O. TANAKa: Ultrastructure of the developing stomach in human embryos. Anat. Embryol. 187: 145-151 (1993).

Pereda, J., H. Cerisola and J. Pozo: El saco vitelino humano: superficie endodermica y mesotelial analizadas por microscopia electronica de barrido. An. Anat. Normal (Santiago, Chile) 6: 90-94 (1987).

REYNolds, E. S.: The use of citrate at high $\mathrm{pH}$ as an electron opaque stain in electron microscopy. J. Cell Biol. 17: 208-212 (1963).

Shi, W. K., B. Hopkins, S. Thompson, J. K. Heath, B. M. LukE and C. F. Graham: Synthesis of apolipoproteins, alphafoetoprotein, albumin, and transferrin by the human foetal yolk sac and other foetal organs. J. Embryol. Exp. Morphol. 85: 191-206 (1985).

Takashina, T.: The significance of human yolk sac (In Japanese). Sanfujinka Chiryo 42: 735-744 (1981).

- Hemopoiesis in the human yolk sac. Amer. J. Anat. 184: 237-244 (1989).

TiedemanN, K.: On the yolk sac of the cat. Endodermal and mesothelium. Cell Tiss. Res. 173: 109-127 (1976).
: On the yolk sac of the cat. II. Erythropoietic phases, ultrastructure of aging primitive erythroblasts, and blood vessels. Cell Tiss. Res. 183: 71-89 (1977).

Ukeshima, A., Y. HaYAshi and T. Fujimoto: Surface morphology of the human yolk sac: Endoderm and mesothelium. Arch. Histol. Jap. 49: 483-494 (1986).

WislockI, G. B. and E. W. DEMPSEY: Electron microscopy of the placenta of the rat. Anat. Rec. 123: 33-57 (1955).

WiTschI, E.: Migration of the germ cells of human embryos from the yolk sac to the primitive gonadal folds. Contr. Embryol. Carneg. Inst. 32: 67-80 (1948).

Dr. Jaime PEREDA

Laboratorio de Embriologia

y Microscopia Electronica

Facultad de Medicina

Universidad de Chile

Casilla 226, Correo 13, San Miguel

Santiago, Chile 